\title{
Abnormalities of the peripheral circulation and respiratory function in patients with severe heart
} failure

\author{
A J COWLEY, K STAINER, J M ROWLEY, J R HAMPTON \\ From the Department of Medicine, University Hospital, Nottingham
}

SUMMARY To investigate the peripheral circulatory and respiratory abnormalities which occur in patients with heart failure, forearm and calf blood flow were measured before and after upright exercise, and respiratory gas exchange was measured during exercise in 26 patients with severe heart failure. Compared with a group of normal subjects the patients had reduced limb blood flow at rest and the response of limb blood flow to upright exercise was also abnormal. The increase in calf blood flow after exercise and the reduction in blood flow in the non-exercising forearm were both smaller in patients than in controls. There was a significant correlation between the maximum exercise duration of the patients and calf blood flow both after exercise and at rest. Compared with another group of control subjects the patients had a higher minute ventilation during exercise and a reduced oxygen consumption. The respiratory exchange ratio during exercise was also higher in patients than in controls. This suggests that there is early onset of anaerobic metabolism during exercise in patients with severe heart failure.

Severe heart failure is characterised by abnormalities in the peripheral circulation. Both arterial and venous vasoconstriction occur which ultimately may be detrimental to the performance of the failing myocardium. Attempts to correct these abnormalities provide the pathophysiological basis for the use of vasodilators in heart failure. ${ }^{1}$ Monitoring of the central haemodynamic effects of vasodilators indicates that many agents have beneficial effects on myocardial performance. ${ }^{2-4}$ There is now considerable evidence, however, that short term changes in central haemodynamics in response to vasodilator treatment do not correlate with symptomatic improvement as judged by changes in exercise tolerance. ${ }^{56} \mathrm{We}$ have previously shown that changes in the arteriolar tone of a vascular bed consisting predominantly of skeletal muscle correlate with changes in exercise tolerance in response to vasodilator treatment. ${ }^{7}$ This suggests that an important effect of vasodilator treatment is to cause arteriolar

Requests for reprints to Dr A J Cowley, Department of Medicine, University Hospital, Nottingham NG7 2UH.

Accepted for publication 14 September 1985 vasodilatation in skeletal muscle and so improve oxygen delivery to that muscle during exercise.

Limb blood flow can be measured by simple noninvasive techniques. Previous reports have suggested that blood flow is reduced in the forearm, a vascular bed consisting predominantly of skeletal muscle, in patients with severe heart failure. ${ }^{8}$ In addition the increase in blood flow in response to ischaemia and isometric exercise is attenuated compared with that in normal subjects. ${ }^{8}$ Little is known, however, about the effect of upright exercise on limb blood flow. To investigate the response to upright exercise we have made simultaneous measurements of forearm and calf blood flow before and after exercise in a group of patients with severe heart failure.

Patients with heart failure have a reduced maximal oxygen consumption during exercise, and blood lactate concentrations indicate that the onset of anaerobic metabolism in exercise occurs earlier than it does in normal subjects. ${ }^{910}$ This implies that oxygen consumption may be below normal during submaximal exercise. To investigate this possibility we have measured oxygen uptake, respiratory exchange ratio, and minute ventilation during exercise and have compared the results obtained with those from a group of normal subjects. 


\section{Patients and methods}

\section{PATIENTS}

Twenty six patients aged 46-69 years were studied; 24 were male. All were limited on exertion by fatigue or dyspnoea or both despite taking at least $80 \mathrm{mg}$ of frusemide daily. Heart failure was due to ischaemic heart disease in 19 of the patients and to dilated cardiomyopathy in the remaining seven. All had been on stable treatment for at least four weeks before being studied and none was taking a vasodilator.

\section{EXERCISE TESTING}

At each visit the patients were exercised on a treadmill to a symptom limited maximum. The speed and slope of the treadmill was increased after three minutes at each of the following stages given as $\mathrm{kph}$ and slope in degrees; I, 2.7 and $0 ; \mathrm{II}, 2.7$ and 1.3 ; III, 2.7 and 2.6 ; IV, 2.7 and $4.0 ; \mathrm{V}, 4.0$ and 5.6 ; VI, 5.4 and $6 \cdot 2$; VII, $6 \cdot 8$ and $7 \cdot 2$.

To overcome changes which may have occurred due to familiarisation with the exercise protocol and with the method used to measure respiratory gases each patient was exercised on consecutive weekly visits until their symptom limited exercise time at two consecutive visits did not increase by more than $5 \%$. This last exercise test was taken to represent the maximum exercise tolerance of the patient, and the measurements of limb blood flow and respiratory function made on that occasion were those that are used in this report.

\section{RESPIRATORY GAS ANALYSIS}

During each symptom limited exercise test oxygen uptake, respiratory exchange ratio, and ventilation were measured every minute with an online gas analyser (PK Morgan).

\section{MEASUREMENT OF LIMB BLOOD FLOW}

Initial measurements of limb blood flow were made before the symptom limited exercise test, and after this exercise test each patient was rested in a temperature controlled room $\left(22-24^{\circ} \mathrm{C}\right)$ for one hour. By this time limb blood flow had returned to preexercise values. Each patient then underwent a second, submaximal, exercise test. A submaximal test was used to determine the effects of exercise on limb blood flow for two reasons. First to enable us to measure the highest oxygen consumption of the patients during the symptom limited test, and second to eliminate movement artefacts from the recordings caused by the excessive ventilatory effort of maximal exercise. The duration of the submaximal test was determined by the patients' performance during the maximal test. Each patient was asked to exercise to achieve a workload that in the symptom limited test required an oxygen consumption of $12 \mathrm{ml} / \mathrm{kg} /$ minute. If the patient failed to achieve this oxygen consumption during the symptom limited test the submaximal test was terminated at the end of the exercise stage before the one in which they became symptom limited. This level of workload was chosen because it represented a moderate degree of exercise for most of the patients and experience had shown that it produced substantial changes in limb blood flow in normal controls.

Simultaneous measurements of forearm and calf blood flow were made in the right forearm and calf by venous occlusion plethysmography using mercury-in-silastic strain gauges. ${ }^{11}$ In brief, the technique consists of measuring the rate of change of limb volume, which is determined by limb blood flow, in response to intermittent venous occlusion. Limb blood flow was measured at rest and every minute from three to 13 minutes after submaximal exercise. The right arm was held loosely at the side during the submaximal exercise test.

\section{CONTROL SUBJECTS}

Two groups of normal subjects without signs or symptoms of cardiovascular disease were used as controls. All the subjects were male. The age range was similar in patients and controls. Eighteen subjects were exercised to the end of stage IV of the protocol during which measurements of oxygen consumption, respiratory exchange ratio, and ventilation were made. Seventeen subjects in a second group were exercised to establish a workload that required an oxygen consumption of 12 $\mathrm{ml} / \mathrm{kg} /$ minute. After one hour's rest forearm and calf blood flow measurements were made at rest and 3-13 minutes after exercise to this workload.

\section{STATISTICAL ANALYSIS}

Analysis of variance for repeated measures was used to compare changes in limb flow between the patients and controls. We used $t$ tests to compare differences in respiratory function during exercise between the patients and the controls, during which the patient number varied. Comparison of calf blood flow with maximum exercise time was performed with Pearson's moment correlation coefficient.

\section{Results}

The mean maximum exercise time for the group of patients was 10.47 minutes (range 1.2-17.5 minutes) and the mean highest oxygen consumption was 12.5 $\mathrm{ml} / \mathrm{kg} /$ minute (range $5.8-25.2 \mathrm{ml} / \mathrm{kg} /$ minute). 


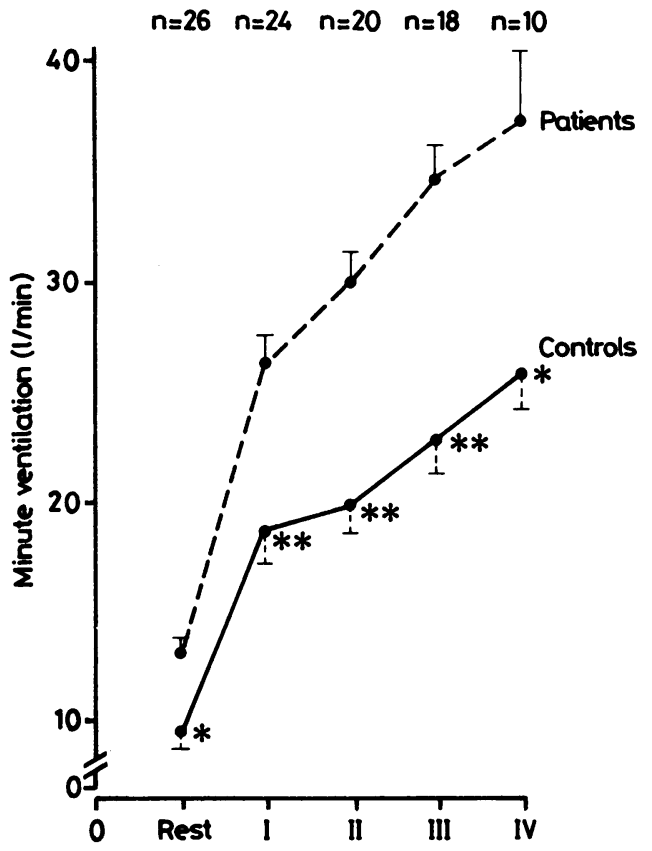

Fig. 1 Mean (SEM) values of minute ventilation at rest and during stages I-IV of the exercise protocol for patients and controls. ${ }^{\star} p<0.05,{ }^{\star \star} p<0.005$.

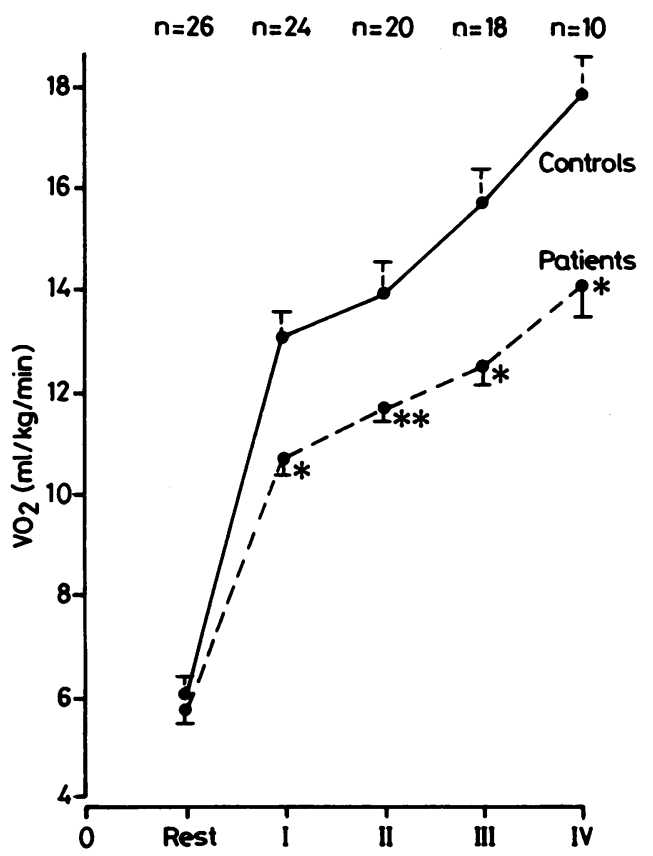

Fig. 2 Mean (SEM) values of oxygen uptake at rest and during stages $I-I V$ of the exercise protocol in patients and controls. ${ }^{\star} p<0.03,{ }^{\star \star} p<0.02$.

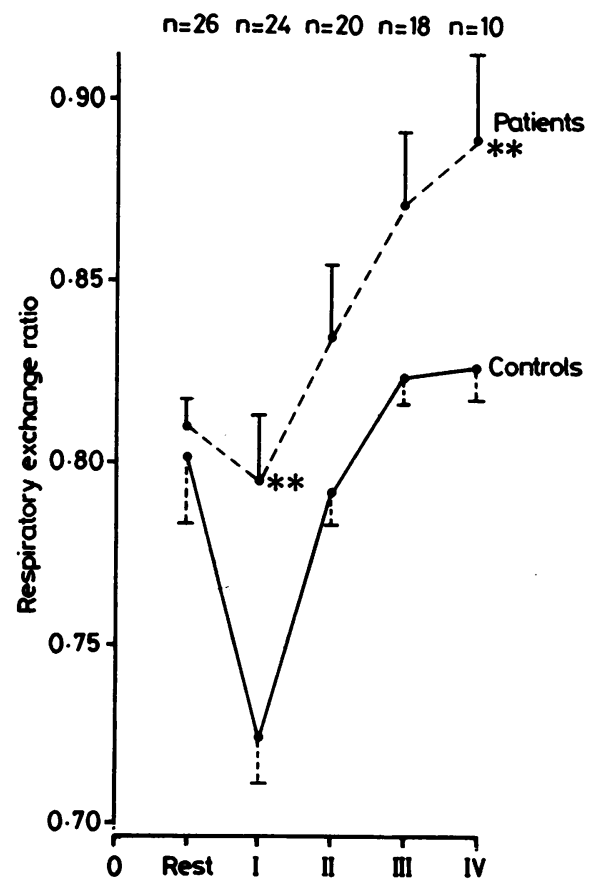

Fig. 3 Mean (SEM) values of respiratory exchange ratio at rest and during stages $I-I V$ of the exercise protocol for patients and controls. ${ }^{\star \star} p<0.05$.

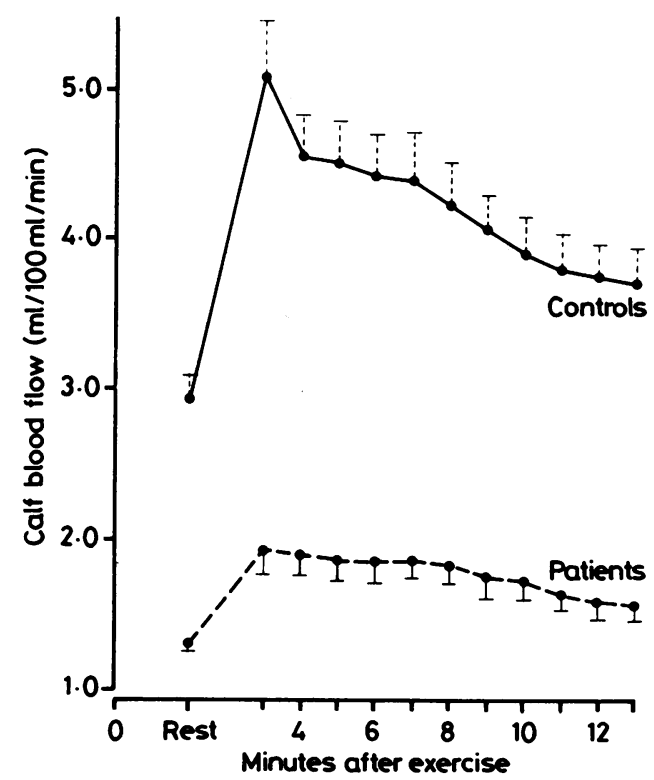

Fig. 4 Mean (SEM) values of calf blood flow at rest and from 3-13 minutes after submaximal exercise for the patients and controls. $p<0.001$. 


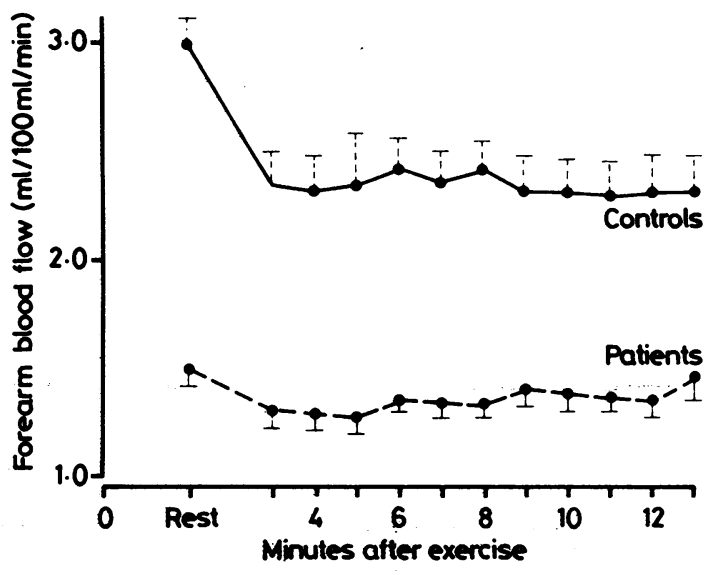

Fig. 5 Mean (SEM) values of forearm blood flow at rest and from 3-13 minutes after submaximal exercise in patients and controls. $p<0.001$.

MEASUREMENTS OF RESPIRATORY FUNCTION

Fig. 1 shows the mean (SEM) values of minute ven- tilation during exercise for the patients and controls. Twenty six patients had resting measurements made, 24 patients completed stage I of the exercise protocol, 20 completed stage II, 18 completed stage III, and 10 completed stage IV. The patients had higher values for minute ventilation both at rest and during exercise than the controls $(p<0.05$ at rest, $p<0.005$ at stage I, p $<0.005$ at stage II, p $<0.005$ at stage, III, and $p<0.05$ at stage IV).

Fig. 2 shows the effects of exercise on the mean (SEM) values of oxygen uptake in the patients and the control subjects. Oxygen consumption was not significantly different between the patients and the control subjects at rest. As exercise progressed there was the expected increase in oxygen consumption in both groups but the patients consistently showed a lower oxygen consumption than the control subjects $(p<0.03$ at stage $I, p<0.02$ at stage II, $p<0.03$ at stage III, and $p<0.03$ at stage IV).

Fig. 3 shows the values for respiratory exchange ratio for the patients and the control subjects. There was no difference between the groups at rest; however, as exercise progressed the patients showed a

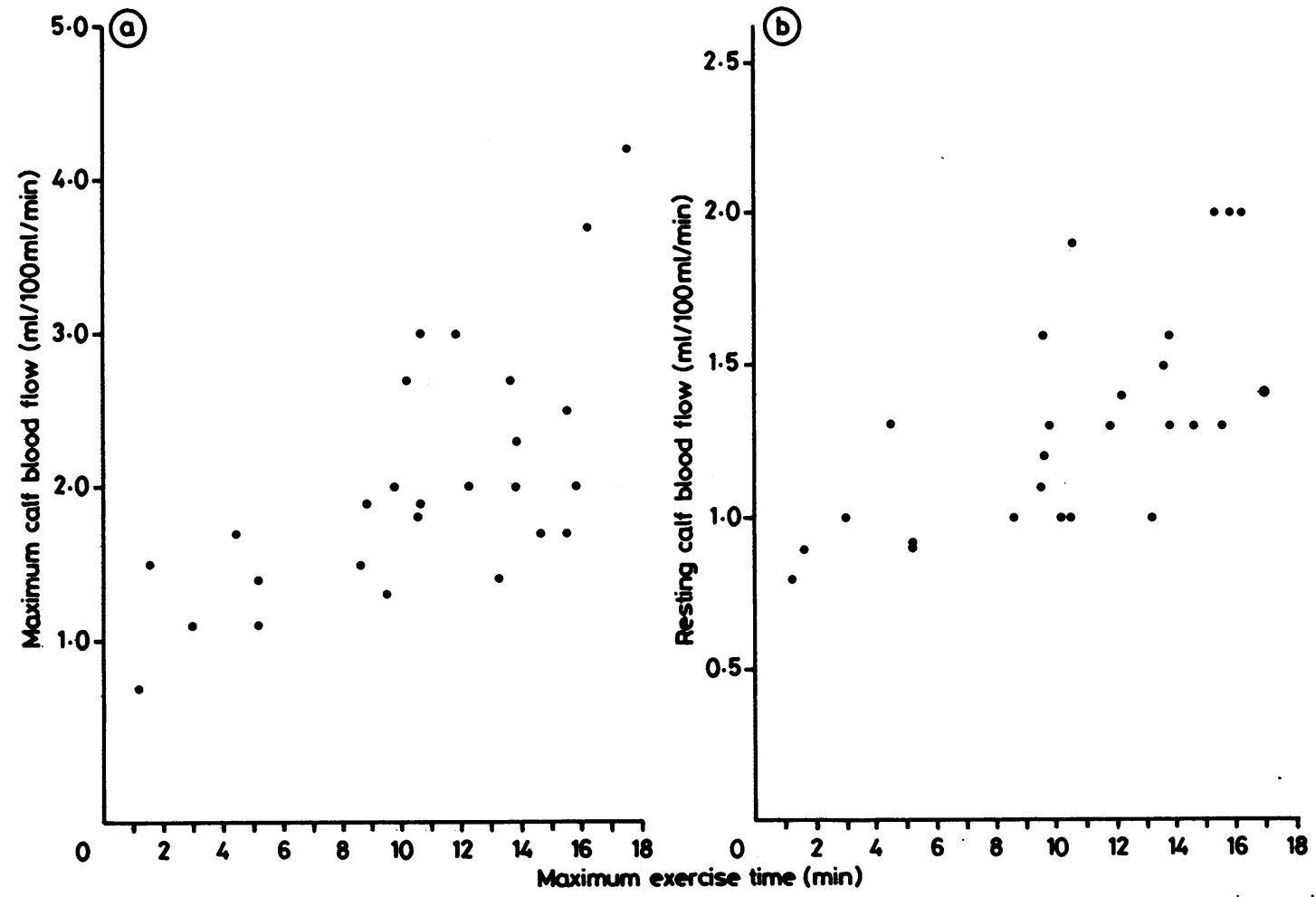

Fig. 6 (a) Relation between maximum exercise time and maximum value of calf blood flow after submaximal exercise in patients. $r=0.642, p<0.01$. (b) Relation between maximum exercise time and resting calf blood flow in patients. $r=0.526$, $p<0.02$. 
higher respiratory exchange ratio than the control subjects ( $p<0.05$ at stage I and stage IV).

\section{MEASUREMENTS OF LIMB BLOOD FLOW}

The mean (SEM) oxygen consumption of the patients at the end of the submaximal exercise test was $11.6( \pm 0.58) \mathrm{ml} / \mathrm{kg} / \mathrm{min}$. Fig. 4 shows the resting and post-exercise values of calf blood flow for the patients and the control subjects. The mean (SEM) value of resting calf blood flow in the control subjects was $2.98( \pm 0.14) \mathrm{ml} / \mathrm{dl} / \mathrm{min}$, whereas in the patients it was $1.32( \pm 0.07) \mathrm{ml} / 100 \mathrm{ml} / \mathrm{min}$. After submaximal exercise calf blood flow had increased in the control subjects to a peak of $5.07( \pm 0.39) \mathrm{ml} / 100$ $\mathrm{ml} / \mathrm{min}$. Peak calf blood flow after exercise in the patients was $1.93( \pm 0.17) \mathrm{ml} / 100 \mathrm{ml} / \mathrm{min}$. The patients, therefore, not only had reduced calf blood flow at rest but also had an attenuated vasodilator response to similar degrees of exercise $(p<0.001)$.

Fig. 5 shows the effect of exercise on blood flow in the non-exercising forearm. The mean (SEM) resting value for the control subjects was $2.98( \pm 0 \cdot 12)$ $\mathrm{ml} / 100 \mathrm{ml} / \mathrm{min}$ compared with a resting value for the patients of $1.51( \pm 0.09) \mathrm{ml} / 100 \mathrm{ml} / \mathrm{min}$. After exercise, forearm blood flow in the controls fell to $2.35( \pm 0.14) \mathrm{ml} / 100 \mathrm{ml} / \mathrm{min}$ three minutes after exercise. The corresponding value in the patients was $1.30( \pm 0.07) \mathrm{ml} / 100 \mathrm{ml} / \mathrm{min}$. The reduction in blood flow in the forearm after exercise was therefore considerably smaller in the patients $(p<0.001)$.

Fig. 6a shows the relation between maximum exercise time and the maximum value of calf blood flow achieved after submaximal exercise for each patient. There was a significant positive correlation between these variables, $r=0.642(p<0.01)$. Resting values of calf blood flow and maximum exercise time were also positively correlated, $r=0.526(p<0.02$, Fig. 6b).

\section{Discussion}

This study has shown that patients with severe heart failure who remain symptomatic despite diuretic treatment have abnormalities of limb blood flow and of respiratory gas exchange. Since one of the predominant symptoms of patients with severe heart failure is dyspnoea it is not surprising that the patients had a higher minute ventilation, both at rest and during exercise, than the controls.

The differences in oxygen consumption are, however, more surprising. There was no difference in oxygen consumption between the patients and the controls at rest, but oxygen consumption was consistently lower in the patients during exercise. This observation does not accord with some published reports. ${ }^{1213}$ Franciosa et al have suggested that oxy- gen consumption during submaximal exercise is the same in patients with heart failure as normal controls. ${ }^{12}$ Six of their patients, however, had a "normal" exercise capacity with a maximal oxygen consumption of greater than $20 \mathrm{ml} / \mathrm{kg} /$ minute, whereas only one of our patients had a maximal oxygen consumption as high as this. Furthermore the maximal oxygen consumption of many of their patients was greater than that in our patients. Similarly 12 of the patients studied by Patterson et al had a mean maximal oxygen consumption of 32.5 $\mathrm{ml} / \mathrm{kg} / \mathrm{min} .{ }^{13}$ The inclusion of these patients in the comparison of oxygen consumption during submaximal exercise with normal controls may well have hidden a reduction in oxygen consumption in the patients with more severe heart failure. Wilson et al measured oxygen consumption during submaximal exercise in patients with varying degrees of heart failure and showed a non-significant trend towards a lower systemic oxygen consumption during submaximal exercise in patients with more severe heart failure. ${ }^{14}$

The higher respiratory exchange ratio for the patients during exercise is consistent with the lower oxygen consumption and indicates the early onset of anaerobic metabolism.

The results described here are supported by studies which evaluated the metabolic consequences of exercise in the limbs in patients with heart failure. Patients with severe heart failure have a lower oxygen consumption in the forearm than controls in response to submaximal exercise. ${ }^{1516}$ In addition differences in the forearm skeletal muscle metabolism between patients and control subjects have been reported in a nuclear magnetic resonance study. ${ }^{17}$ These data suggest that there is a reduction in oxygen consumption in skeletal muscle during submaximal exercise in patients with heart failure; this must be reflected in a reduction of systemic oxygen consumption, as we have demonstrated.

The differences in limb blood flow between the patients and controls may explain the differences in oxygen consumption. Patients with heart failure are capable of a greater oxygen extraction than controls. ${ }^{16}$ This compensatory mechanism may, however, be insufficient to prevent the early onset of anaerobic metabolism if blood flow to exercising muscle is severely reduced. This study has demonstrated that both forearm and calf blood flow are reduced at rest in patients with heart failure. Although the technique we used to measure limb blood flow does not permit us to make measurements during exercise, the changes that occurred after exercise allow us to make some assumptions about changes during exercise. The increase in calf blood flow after exercise was considerably attenuated in the 
patients compared with that in the controls and, therefore, it is highly likely that calf blood flow, and hence oxygen delivery to the exercising skeletal muscle, was reduced during exercise. The changes in limb blood flow in the non-exercising forearm indicate that the resistance vessels in the forearm were near maximally constricted at rest and were unable to constrict further during exercise to aid redistribution of blood to the exercising limb.

The relation between resting and post-exercise calf blood flow and maximum exercise capacity of the patients indicates that limb blood flow is a major determinant of exercise tolerance in patients with severe heart failure. Since fatigue on exertion is also a major symptom of patients and is due to lactate accumulation in skeletal muscle and the associated intracellular acidosis,,${ }^{14}$ the extent of the reduction in blood flow would determine the onset of these changes during exercise. Using different techniques others have also suggested that reduced limb blood flow is an important cause of the reduced exercise tolerance of patients with severe heart failure. ${ }^{14}$

The reduced blood flow to exercising skeletal muscle would also account for the lack of immediate improvement in exercise tolerance in patients treated with vasodilators, despite immediate improvement in central haemodynamics. It has been shown that in the short term captopril improves central haemodynamics without affecting exercise time. ${ }^{6} \mathrm{We}$ and others have demonstrated that short term administration of captopril does not affect limb blood flow and it is only after more prolonged administration that limb blood flow improves. ${ }^{78}$ Thus the improvement in exercise time seems to be related more to the improved blood flow to skeletal muscle than to central haemodynamic effects.

Comparison of the changes in the peripheral circulation and respiratory gas exchange in patients with severe heart failure with those in normal subjects indicates some of the important pathophysiological changes that occur in patients with heart failure. The techniques used in this study are non-invasive and readily repeatable and may therefore be used to assess the response of patients to treatment.

\section{References}

1 Cohn JN. Physiologic basis for vasodilator therapy for heart failure. $A m \mathcal{F}$ Med 1981; 71: 135-9.

2 Maslowski AH, Nicholls MG, Ikram H, Espiner EA. Haemodynamic, hormonal and electrolyte responses to captopril in resistant heart failure. Lancet 1981; i: 71-4.

3 Fitchett DH, Neto JAM, Oakley CM, Goodwin JF. Hydralazine in the management of left ventricular fail- ure. Am $\mathcal{f}$ Cardiol 1979; 44: 303-9.

4 Williams DO, Bommer WJ, Miller RR, Amsterdam EA, Mason DT. Haemodynamic assessment of oral peripheral vasodilator therapy in chronic congestive heart failure: prolonged effectiveness of isosorbide dinitrate. Am $\mathcal{F}$ Cardiol 1977; 39: 84-9.

5 Franciosa JA, Park M, Levine TB. Lack of correlation between exercise capacity and indexes of resting left ventricular performances in heart failure. $\mathrm{Am} \mathcal{F} \mathrm{Cardiol}$ 1981; 47: 33-9.

6 Massie BM, Krammer BL, Topic N. Lack of relationship between the short-term hemodynamic effects of captopril and subsequent clinical responses. Circulation 1984; 69: 1135-41.

7 Cowley AJ, Rowley JM, Stainer K, Hampton JR. Captopril therapy for heart failure: a placebo controlled study. Lancet 1982; ii: 730-2.

8 Mason DT, Zelis R, Longhurst J, Lee G. Cardiocirculatory response to muscular exercise in congestive heart failure. Prog Cardiovasc Dis 1977; 19: 475-89.

9 Rubin SA, Chatterjee K, Parmley WW. Metabolic assessment of exercise in chronic heart failure patients treated with short-term vasodilators. Circulation 1980; 61: 543-8.

10 Huckabee WE, Judson WE. The role of anaerobic metabolism in the performance of mild muscular work. Relationship to oxygen consumption and cardiac output and the effects of congestive cardiac failure. $\mathcal{F}$ Clin Invest 1958; 37: 1577-602.

11 Greenfield ADM, Whitney RI, Mowbray FR. Methods for the investigation of peripheral blood flow. $\mathrm{Br} \mathrm{Med}$ Bull 1963; 19: 101-4.

12 Franciosa JA, Ziesche $S$, Wilen $M$. Functional capacity of patients with chronic left ventricular failure. $A m \mathcal{F}$ Med 1976; 67: 460-6.

13 Patterson JA, Naughton J, Dietras RJ, Gunnar RM. Treadmill exercise in assessment of the functional capacity of patients with cardiac disease. $\mathrm{Am} \mathfrak{f}$ Cardiol 1972; 30: 757-62.

14 Wilson JR, Martin JL, Schwarty D, Ferraro N. Exercise intolerance in patients with chronic heart failure: role of impaired nutritive flow to skeletal muscle. Circulation 1984; 69: 1079-87.

15 Zelis R, Longhurst J, Capone RJ, Mason DT. A comparison of regional blood flow and oxygen utilization during dynamic forearm exercise in normal subjects and patients with congestive heart failure. Circulation 1974; 50: $137-43$.

16 Longhurst J, Gifford W, Zelis R. Impaired forearm oxygen consumption during static exercise in patients with congestive heart failure. Circulation 1976; 54: 477-9.

17 Wilson JR, Fink L, Mavis J, et al. Evaluation of energy metabolism in skeletal muscle of patients with heart failure with gated phosphorus-31 nuclear magnetic resonance. Circulation 1985; 71: 57-62.

18 Faxon DP, Halperin JL, Craeger MA, Gavras H, Schlek EC, Ryan TJ. Angiotensin inhibition in severe heart failure: acute central and limb haemodynamic effects of captopril with observations on sustained therapy. Am Heart $\mathcal{F}$ 1981; 101: 548-56. 